\title{
Effects of Additive Type on Fermentation and Aerobic Stability and Its Interaction with Air Exposure on Silage Nutritive Value
}

\author{
Horst Auerbach ${ }^{1, *(D)}$ and Elisabet Nadeau ${ }^{2,3}$ (D) \\ 1 International Silage Consultancy, Thomas-Müntzer-Strasse 12, 06193 Wettin-Löbejün, Germany \\ 2 Department of Animal Environment and Health, Swedish University of Agricultural Sciences (SLU), \\ P.O. Box 234, 53223 Skara, Sweden; elisabet.nadeau@slu.se \\ 3 Research \& Development, The Rural Economy and Agricultural Society Sjuhärad, P.O. Box 5007, \\ 51405 Länghem, Sweden \\ * Correspondence: horst.uwe.auerbach@outlook.de; Tel.: +49-1520-1683167
}

Received: 23 July 2020; Accepted: 17 August 2020; Published: 20 August 2020

\begin{abstract}
As farm profitability and sustainability of animal production are largely affected by overall losses of dry matter and nutritive value of silage from field to trough, the objective of the study was to assess the effects of different additive types on fermentation, aerobic stability (ASTA) and changes in in vitro organic matter digestibility (IVOMD) and metabolisable energy (ME) of grass and grass-clover silage exposed to air. Three trials were performed, where grass and grass-clover forages were treated with biological and chemical additives and ensiled in 1.6-L glass jars. Upon silo opening, fermentation characteristics, yeast counts and ASTA were determined as well as changes in IVOMD and ME during subsequent air exposure for up to $336 \mathrm{~h}$. All silages were well preserved. The ASTA was improved by Lactobacillus buchneri-containing additives in all trials and by chemical additives in trial 3. In untreated silage, aeration reduced IVOMD and ME but variable effects of additives were observed. The nutritive value was maintained throughout aeration by all additives in trial 1 , whereas in trial 3, only chemical additives were successful. A strong negative linear relationship across trials was detected between the extent of aerobic deterioration and changes in ME during air exposure $(r=-0.756, p<0.001)$. Silage additives improving aerobic stability have the potential to prevent the loss of nutritive value of grass and grass-clover silage during feed-out.
\end{abstract}

Keywords: aerobic deterioration; digestibility; fermentation characteristics; grass silage; nutritive value; silage additives; yeasts

\section{Introduction}

Silage from various crops plays an important role in ruminant nutrition in many geographical areas of the world. Variations in silage quality and losses from field to trough largely affect the environmental impact of animal production as well as profitability and sustainability [1-4]. Therefore, all metabolic pathways facilitated by undesired microorganisms, which lead to significant losses of nutrients and energy during storage, e.g., butyric acid fermentation by clostridia and ethanol production by yeasts $[1,5]$, must be suppressed in order to maintain the highest silage quality until silo opening. Furthermore, additional losses of up to $20 \%$ of the stored dry matter (DM) and a reduction in nutritive value during the aerobic phase after silo opening may be incurred by the activity of yeasts, and other aerobic microorganisms, e.g., moulds [1]. In addition to DM losses, deteriorating silage and total-mixed rations can cause further indirect economic losses by decreased feed intake and performance [6-8], or by adverse effects on animal health due to the formation of mycotoxins $[9,10]$. 
Apart from good silage management practices, silage additives have an important role in mitigating the detrimental effects of undesired silage microorganisms on fermentation and nutritive value as well as on aerobic stability (ASTA) [11,12]. Based on an evaluation of numerous silage studies, homofermentative lactic acid bacteria $\left(\mathrm{LAB}_{\mathrm{ho}}\right)$ improved the efficiency of the fermentation process of grass by increasing DM recovery, or reduced DM losses [12,13]. Although Oliveira et al. [13] detected no effect of $\mathrm{LAB}_{\mathrm{ho}}$ inoculation on ASTA across a range of different silage types, others have shown that $\mathrm{LAB}_{\text {ho }}$ may frequently impair aerobic stability $[14,15]$. Positive effects of $L A B_{\text {ho }}$ on grass silage digestibility have been reported but they were highly variable [16-18]. The meta-analysis by Oliveira et al. [13] did not reveal any improvements in digestibility or DM intake of silage made from grasses and legumes but showed higher milk production by inoculation with LAB. Obligately heterofermentative lactic acid bacteria $\left(\mathrm{LAB}_{\mathrm{he}}\right)$, with Lactobacillus buchneri being the most widely used species in commercial additive preparations, have been shown to enhance silage aerobic stability by producing antifungal acetic acid $[14,19]$. Especially in grass silage, treatment with dual-purpose inoculants combining $\mathrm{LAB}_{\text {ho }}$ and $\mathrm{LAB}_{\text {he }}$ proved successful in improving the fermentation process and to alleviate the detrimental effects of the sole use of $\operatorname{LAB}_{\text {ho }}$ on aerobic stability $[20,21]$. Formic acid and their salts as well as sodium nitrite in combination with hexamethylene tetramine or calcium formate, which have mainly been used in low DM grasses and legumes, have the potential to improve fermentation quality and animal performance [11,12,22-24], but they usually have no effect on aerobic stability in low DM silages [14,15]. Salts of antifungal sorbic, benzoic and propionic acids, applied alone or in combination, have been shown to restrict, or completely prevent, silage deterioration for extended periods of exposure to air [15,25-27]. In general, only very limited experimental data is available on the effects of aeration on changes in nutritive value in maize, sorghum and whole-crop wheat silage [28-30], and to our knowledge, no study has yet addressed this topic in grass and grass-clover silage.

Due to scarcity of comparative data, the first aim of this study was to broaden the knowledge on the effect of biological and chemical additives of different compositions on fermentation pattern, yeast count and aerobic stability of grass and grass-clover silage. Secondly, changes in nutritive value of grass and grass-clover silage during air exposure after silo opening were monitored. We hypothesised that the feed value of grass and grass-clover silage determined at silo opening can be maintained during feed-out by preventing heating of the silage through the use of silage additives with expected mode of action against aerobic deterioration.

\section{Materials and Methods}

\subsection{Ensiling}

Forages from the first regrowth, which were obtained from dairy farms near Skara, south-west Sweden ( $58^{\circ} \mathrm{N} \mathrm{29^{ \prime }}$ E), composed of a mixture of timothy (Phleum pratense L.) and meadow fescue (Festuca pratensis L.) in trial 1 and of a mixture of timothy, meadow fescue and red clover (Trifolium pratense L.; $80-90 \%$ grass $/ 10-20 \%$ red clover of DM) in trials 2 and 3. Forages were harvested on July 17 and July 18, 2016 in trials 2 and 1, respectively, and on 4 July 2017 in trial 3, and wilted in the field overnight. In each trial, wilted forage was collected from different locations in the field, chopped to $20 \mathrm{~mm}$ theoretical particle length by using a stationary chopper and finally mixed well and composited to have homogeneous material for the ensiling trial. Chemical compositions of the forages before additive application are shown in Table 1. 
Table 1. Composition of wilted forages before additive application.

\begin{tabular}{cccccccc}
\hline \multirow{2}{*}{ Trial } & DM & Crude Ash & Crude Protein & aNDFom ADFom & ADL & WSC \\
\cline { 2 - 7 } & $\mathbf{g ~ k g}^{-1}$ & 90 & 112 & 549 & 306 & 28 & 138 \\
\hline 1 & 419 & 108 & 128 & 578 & 322 & 39 & 106 \\
2 & 351 & 195 & 462 & ND & ND & 108 \\
3 & 216 & 88 & $\mathbf{~} \mathbf{~ D M ~}$ \\
\hline
\end{tabular}

DM, dry matter; aNDFom, neutral-detergent fibre excluding ash with $\alpha$-amylase addition; ADFom, acid-detergent fibre excluding ash; ADL, acid-detergent lignin; WSC, water-soluble carbohydrates; ND not determined. Analyses performed on one composite sample per trial.

The following additive treatments were applied manually as aqueous suspensions (biological additives) or dilutions (chemical additives) in tap water to give an application volume of $10 \mathrm{~mL} \mathrm{~kg}$-1 herbage: untreated control, treated with tap water $(\mathrm{CON})$; $\mathrm{LAB}_{\text {he }}$ inoculant solely composed of Lactobacillus buchneri CNCM-I 4323 at an inoculation rate of $1.0 \times 10^{5} \mathrm{cfu}^{-1}$; dual-purpose inoculant containing a combination of $\mathrm{LAB}_{\text {he }}$ und $\mathrm{LAB}_{\text {ho }}$ composed of Lactobacillus buchneri CNCM-I 4323 and Pediococcus acidilactici DSM 11673 at a total inoculation rate of $1.67 \times 10^{5} \mathrm{cfu} \mathrm{g}^{-1}\left(\mathrm{LAB}_{\text {heho }}\right)$, and a liquid chemical mixture (NHS) containing sodium nitrite $\left(195 \mathrm{~g} \mathrm{~L}^{-1}\right)$, hexamethylene tetramine $\left(71 \mathrm{~g} \mathrm{~L}^{-1}\right)$ and potassium sorbate $\left(106 \mathrm{~g} \mathrm{~L}^{-1}\right)$, added at $2.5 \mathrm{~mL} \mathrm{~kg}^{-1}$ in trial 1 and at $2.0 \mathrm{~mL} \mathrm{~kg}^{-1}$ in trials 2 and 3 In addition, the following additive treatments were studied in trial 3: $\mathrm{LAB}_{\text {ho }}$ inoculant composed of Lactobacillus plantarum DSM 16627 and Lactobacillus paracasei NCIMB 30151 at a total inoculation rate of $1.5 \times 10^{5} \mathrm{cfu} \mathrm{g}^{-1}$ and a liquid chemical mixture (BNP) containing sodium benzoate $\left(238 \mathrm{~g} \mathrm{~L}^{-1}\right)$, sodium nitrite $\left(119 \mathrm{~g} \mathrm{~L}^{-1}\right)$ and sodium propionate $\left(12 \mathrm{~g} \mathrm{~L}^{-1}\right)$ at $2 \mathrm{~mL} \mathrm{~kg}^{-1}$. All additives were kindly provided by KONSIL Europe GmbH, Wettin-Löbejün, Germany. Treated silage was manually packed into 1.6-L glass jars (Weck, Öfingen, Germany) and closed with rubber O-ring, glass lid and spring clips. To facilitate air ingress, jars had a hole $(6 \mathrm{~mm}$ diameter) in the body (about $30 \mathrm{~mm}$ above the bottom) and in the lid, which were closed by rubber stoppers. During the 56-day storage period at 20-22 ${ }^{\circ} \mathrm{C}$, these rubber stoppers were removed for $24 \mathrm{~h}$ on day 28 and 49 . Low packing densities (trial 1: $172 \pm 1.8 \mathrm{~kg} \mathrm{DM} \mathrm{m}^{-3}$, trial 2: $153 \pm 3.9 \mathrm{~kg} \mathrm{DM} \mathrm{m}^{-3}$, trial 3: $118 \pm 0.5 \mathrm{~kg} \mathrm{DM} \mathrm{m}^{-3}$ ) were chosen to enable free air circulation through the silage when rubber stoppers were removed. Three replicate silos were used for each additive treatment in a completely randomised design.

\subsection{Laboratory Analysis}

The DM concentration of wilted forage and silage was determined by oven-drying at $60^{\circ} \mathrm{C}$ until constant weight, followed by $3 \mathrm{~h}$ at $105^{\circ} \mathrm{C}$. Silage DM was corrected for the loss of volatiles during drying [31]. Losses of DM during fermentation were calculated using the equation by Weissbach [32]. Crude ash was determined in a muffle furnace at $525{ }^{\circ} \mathrm{C}$ for $16 \mathrm{~h}$. The total $\mathrm{N}$ concentration of the herbage was analysed according to Kjeldahl and crude protein $(\mathrm{CP})$ was calculated as total $\mathrm{N} \times 6.25$. The ash-free aNDFom, ADFom and ADL were determined on dried $\left(60^{\circ} \mathrm{C}, 24 \mathrm{~h}\right)$ and ground samples by the FibreTech method according to van Soest et al. [33], including $\alpha$-amylase in the NDF analysis but without sodium sulphite. Silage samples were stored at $-18{ }^{\circ} \mathrm{C}$ until analysis. Extracts were prepared by blending $50 \mathrm{~g}$ of silage with $200 \mathrm{~mL}$ of distilled water. After addition of $1 \mathrm{~mL}$ of toluene, the extract was stored overnight at $4{ }^{\circ} \mathrm{C}$. Subsequently, extracts were filtered through a paper filter and a microfilter $(0.45 \mu \mathrm{m})$. Silage $\mathrm{pH}$ was measured by using a calibrated $\mathrm{pH}$-meter (Methrom, Herisau, Switzerland), and ammonia-N concentration was analysed by a colorimetric method based on the Berthelot-reaction (CFA, Scan++, Skalar Analytical, Breda, The Netherlands). Lactic acid was determined by HPLC coupled with a refraction index detector as described by Weiss and Kaiser [34], whereas volatile fatty acids and alcohols were analysed by GC and flame ionisation detection [35]. Water-soluble carbohydrates were determined by the anthrone-method as described by von Lengerken and Zimmermann [36]. 
The count of yeasts was enumerated after preparation of 10-fold serial dilutions of silage samples by using peptone water broth $\left(1 \mathrm{~g} \mathrm{~L}^{-1}\right)$ followed by spread-plating on dichloran-rose bengal chloramphenicol agar (DRBC) and incubation at $25^{\circ} \mathrm{C}$ for 5 days [37]. Aerobic stability was evaluated by the temperature method [38]. Data loggers (Tinytag Talk 2, Gemini, Chichester, UK) were placed in the geometric centre of a plastic container loosely filled with silage to record silage temperature at 2-h intervals. Each plastic container was stored in an insulating polystyrene box with free air circulation for $276 \mathrm{~h}$ (trial 1), $288 \mathrm{~h}$ (trial 2), or $336 \mathrm{~h}$ (trial 3). Room temperature was kept at $20.9 \pm 0.3^{\circ} \mathrm{C}$ (trials 1 and 2 ) and $20.1 \pm 0.6^{\circ} \mathrm{C}$ in trial 3, respectively. Aerobic stability was defined as the time that elapsed until silage temperature increased by more than $2{ }^{\circ} \mathrm{C}$ above ambient temperature. To assess the extent of aerobic deterioration, the cumulated temperature (TCUM) was calculated by adding up the differences between silage and room temperature according to Tabacco et al. [30], who recorded the temperature at 1-h intervals.

Silage samples taken at silo opening before aeration and silage samples taken after aeration were analysed for in vitro organic matter digestibility (IVOMD) by the VOS method where $0.5 \mathrm{~g}$ of dried $\left(60{ }^{\circ} \mathrm{C}, 24 \mathrm{~h}\right)$ and ground sample was incubated in $49 \mathrm{~mL}$ buffer and $1 \mathrm{~mL}$ rumen fluid at $38^{\circ} \mathrm{C}$ for 96 h. Incubation residue was filtered, dried and combusted to determine the digestibility coefficient of organic matter. Rumen fluid was collected from a fistulated non-lactating dairy cow fed a standard diet of hay, straw and concentrate at maintenance level [39,40]. The content of metabolisable energy (ME) was calculated using the VOS value [41].

\subsection{Calculations and Statistical Analysis}

Fungal counts were $\log _{10}$-transformed before being used in the statistical analysis, and values below the limit of detection of $100 \mathrm{cfu} \mathrm{g}^{-1}$ were set at half of the detection limit (50 $\mathrm{cfu} \mathrm{g}^{-1}=\log _{10} 1.7$ $\mathrm{cfu}^{-1}$ ). If silages were stable over the entire period of air exposure, the hours after which the test was terminated, was used. All data were subjected to ANOVA by employing the procedure MIXED of SAS 9.4 (Cary, NC, USA). A completely randomised design was used with silo as the experimental unit according to the following model: $y_{i j}=\mu+\alpha_{i}+\varepsilon_{i j}$, where $y_{i j}$ is the dependent variable, $\mu$ is the overall mean, $\alpha_{i}$ is the fixed effect of treatment ( $i=1$ to 4 for trials 1 and $2 ; i=1$ to 6 for trial 3 ) and $\varepsilon_{i j}$ is the random error. The model for analysis of the IVOMD and ME values of silages before and after aeration with repeated measurement on replicate nested within treatment was as follows $y_{i j k}=\mu+\alpha_{i}+r_{j}+$ $(\alpha \mathrm{r})_{\mathrm{ij}}+\varepsilon_{\mathrm{ijk}}$ where $\mathrm{y}_{\mathrm{ijk}}$ is the dependent variable, $\mu$ is the overall mean, $\alpha_{\mathrm{i}}$ is the fixed effect of treatment ( $i=1$ to 4 for trials 1 and $2 ; i=1$ to 6 for trial 3 ), $r_{j}$ is the fixed effect of aeration ( $j=1$ to 2 ) and $\varepsilon_{i j k}$ is the random error with compound symmetry as the correlation structure. When the global $F$-test showed significance at $p<0.05$, the Tukey's test was used for pairwise comparisons between least-square means (LSmeans). Trends were reported at $0.05 \leq p<0.10$. The procedure CORR of SAS 9.4 (Cary, NC, USA) was used for the determination of the Pearson partial correlation coefficient, with consideration to the effects of trial and additive treatment, in the relationship between changes in ME concentrations of the silage and the cumulated temperature during aeration. The procedure REG of SAS 9.4 was employed to estimate the slope of the line, the $p$-value and the root mean square error (RMSE) for this relationship and for the relationships between the TCUM and the concentrations of lactic acid plus WSC and that between TCUM and ASTA.

\section{Results}

\subsection{Trial 1}

All silages were well fermented, as reflected by the complete absence of butyric acid, indicating that there was no clostridial activity (Table 2). Losses of DM during fermentation were lowest in NHS treated silage, and the sole use of Lactobacillus buchneri resulted in the highest losses $(p<0.001)$. The NHS application resulted in grass silage with the highest WSC content and the lowest ammonia-N concentration, whereas the opposite was true for $\mathrm{LAB}_{\text {he }}$ treatment $(p<0.001)$. Silage inoculated with 
$\mathrm{LAB}_{\text {he }}$ had larger acetic acid concentrations than any other treatment $(p=0.004)$ and higher ethanol production than $\mathrm{LAB}_{\text {heho }}$ and NHS treatments $(p=0.006)$. Ethanol content tended to be decreased by NHS when compared with untreated silage $(p=0.068)$. In addition, lactic acid was lowest $(p<0.001)$ and $\mathrm{pH}$ was highest $(p<0.001)$ in $\mathrm{LAB}_{\text {he }}$ treated silage. Inoculation with $\mathrm{LAB}_{\text {he }}$ and $\mathrm{LAB}_{\text {heho }}$ led to the formation of larger quantities of 1,2-propanediol compared with silage left untreated or treated with NHS $(p<0.001)$. Other fermentation products, e.g., propionic acid and n-propanol, were not detected. High yeast numbers were enumerated in untreated silage $\left(\log _{10} 6.4 \mathrm{cfu} / \mathrm{g}\right)$, which were substantially reduced to $\log _{10} 3.3 \mathrm{cfu} \mathrm{g}^{-1}$, or lower, by Lactobacillus buchneri-containing inoculants $(p=0.004)$. Concurrently, aerobic stability (ASTA) was lowest in silage, which had not received additive treatment $(p=0.008)$. Inoculated silages were stable over the entire period of exposure to air of $276 \mathrm{~h}$ $(p<0.001)$, and a tendency for increased aerobic stability over that of untreated silage was observed for treatment NHS $(p=0.073)$. The extent of aerobic deterioration as reflected by cumulated temperature was reduced by all additives $(p=0.002)$.

Table 2. Fermentation characteristics, yeast count and aerobic stability of grass silage in trial 1 (LSmeans and SEM in $\mathrm{g} \mathrm{kg}^{-1}$ dry matter unless stated otherwise, $n=3$ ).

\begin{tabular}{ccccccc}
\hline Parameter & CON & LAB $_{\text {he }}$ & LAB $_{\text {heho }}$ & NHS & SEM & $p$ \\
\hline DM $\left(\mathrm{g} \mathrm{kg}^{-1}\right)$ & $407^{\mathrm{b}}$ & $410^{\mathrm{a}, \mathrm{b}}$ & $413^{\mathrm{a}}$ & $410^{\mathrm{a}, \mathrm{b}}$ & 1.4 & 0.026 \\
DM loss (\%) & $5.0^{\mathrm{c}}$ & $6.6^{\mathrm{a}}$ & $5.3^{\mathrm{b}}$ & $4.1^{\mathrm{d}}$ & 0.03 & $<0.001$ \\
WSC & $74.1^{\mathrm{b}}$ & $17.9^{\mathrm{d}}$ & $30.8^{\mathrm{c}}$ & $91.2^{\mathrm{a}}$ & 1.71 & $<0.001$ \\
$\mathrm{NH}_{3} \mathrm{~N}\left(\mathrm{~g} \mathrm{~kg}^{-1} \mathrm{~N}\right)$ & $74^{\mathrm{b}}$ & $82^{\mathrm{a}}$ & $68^{\mathrm{c}}$ & $54^{\mathrm{d}}$ & 0.5 & $<0.001$ \\
pH & $4.16^{\mathrm{c}}$ & $4.31^{\mathrm{a}}$ & $4.07^{\mathrm{d}}$ & $4.24^{\mathrm{b}}$ & 0.005 & $<0.001$ \\
Lactic Acid & $43.4^{\mathrm{b}}$ & $30.5^{\mathrm{d}}$ & $46.7^{\mathrm{a}}$ & $37.8^{\mathrm{c}}$ & 0.47 & $<0.001$ \\
Acetic Acid & $11.0^{\mathrm{b}}$ & $21.0^{\mathrm{a}}$ & $14.2^{\mathrm{b}}$ & $11.0^{\mathrm{b}}$ & 1.43 & 0.004 \\
Propionic Acid & $\mathrm{ND}$ & $\mathrm{ND}$ & $\mathrm{ND}$ & $\mathrm{ND}$ & & \\
Butyric Acid & $\mathrm{ND}$ & $\mathrm{ND}$ & $\mathrm{ND}$ & $\mathrm{ND}$ & & \\
Ethanol & $5.9^{\mathrm{a}, \mathrm{b}}$ & $8.6^{\mathrm{a}}$ & $2.4^{\mathrm{b}}$ & $1.1^{\mathrm{b}}$ & 1.14 & 0.006 \\
n-propanol & $\mathrm{ND}$ & $\mathrm{ND}$ & $\mathrm{ND}$ & $\mathrm{ND}$ & & \\
1,2-propanediol & $4.1^{\mathrm{b}}$ & $14.3^{\mathrm{a}}$ & $9.9^{\mathrm{a}}$ & $3.2^{\mathrm{b}}$ & 1.12 & $<0.001$ \\
Yeast Count (log cfu g & $\left.6.4^{\mathrm{a}}\right)$ & $2.2^{\mathrm{b}}$ & $3.3^{\mathrm{b}}$ & $4.3^{\mathrm{a}, \mathrm{b}}$ & 0.57 & 0.004 \\
ASTA (hours) & $44^{\mathrm{b}}$ & $>276^{\mathrm{a}}$ & $>276^{\mathrm{a}}$ & $201^{\mathrm{a}, \mathrm{b}}$ & 37.8 & 0.008 \\
TCUM $\left({ }^{\circ} \mathrm{C}\right)$ & $786^{\mathrm{a}}$ & $40^{\mathrm{b}}$ & $123^{\mathrm{b}}$ & $301^{\mathrm{b}}$ & 96.0 & 0.002 \\
\hline
\end{tabular}

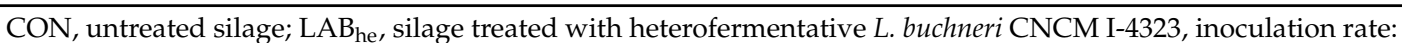
$1 \times 10^{5} \mathrm{cfu} \mathrm{g}^{-1}$; $\mathrm{LAB}_{\text {heho, }}$ silage treated with a mixture of heterofermentative L. buchneri CNCM I-4323 and homofermentative P. acidilactici DSM 11673, total inoculation rate: $1.67 \times 10^{5} \mathrm{cfu} \mathrm{g}^{-1}$; NHS, aqueous mixture of sodium nitrite $\left(195 \mathrm{~g} \mathrm{~L}^{-1}\right)$, hexamethylene tetramine $\left(71 \mathrm{~g} \mathrm{~L}^{-1}\right)$, potassium sorbate $\left(106 \mathrm{~g} \mathrm{~L}^{-1}\right)$, applied at $2.5 \mathrm{~mL} \mathrm{~kg}^{-1}$; SEM, standard error of the mean; WSC, water-soluble carbohydrates; ND, not detected; ASTA, aerobic stability, > denotes that silages were still stable upon termination of the ASTA test after $276 \mathrm{~h}$ of air exposure; TCUM, cumulated temperature during air exposure; ${ }^{\mathrm{a}, \mathrm{b}, \mathrm{c}, \mathrm{d}}$ Least-square (LS) means in rows bearing unlike letters differ at $p<0.05$ (Tukey's test).

Upon silo opening before aeration, no differences in IVOMD and ME among treatments were detected (Table 3). However, during exposure to air, IVOMD of untreated silage declined by 6.9 percentage units, whereas additive use prevented any reduction in $\operatorname{IVOMD}(p<0.001)$. After aeration, the IVOMD of treated silage was 7.2 percentage units higher than that of untreated silage. Concurrently, aeration caused ME concentration to decline by 1.2 megajoule (MJ) $\mathrm{kg}^{-1} \mathrm{DM}$ in untreated silage, but no changes occurred when silage was treated with additives regardless of additive type $(p<0.001)$. The ME concentration after aeration was, on average, $1.2 \mathrm{MJ} \mathrm{kg}^{-1} \mathrm{DM}$ higher in additive-treated than in untreated silage. 
Table 3. In vitro organic matter digestibility (IVOMD, \% of OM) and metabolisable energy (MJ kg-1 $\mathrm{DM})$ concentration of grass silage in trial 1 upon silo opening and after $276 \mathrm{~h}$ of aeration as affected by silage additive (LSmeans and SEM, $n=3$ ).

\begin{tabular}{ccccccc}
\hline Parameter & CON & LAB $_{\text {he }}$ & LAB $_{\text {heho }}$ & NHS & SEM & $p$ \\
\hline \multicolumn{7}{c}{ IVOMD (\% of OM) } \\
\hline Before Aeration & $85.0^{\mathrm{a}}$ & $82.6^{\mathrm{a}}$ & $84.3^{\mathrm{a}}$ & $84.3^{\mathrm{a}}$ & 0.81 & $<0.001$ \\
After Aeration & $78.1^{\mathrm{b}}$ & $85.2^{\mathrm{a}}$ & $84.9^{\mathrm{a}}$ & $85.8^{\mathrm{a}}$ & & \\
Treatment Mean & $81.6^{\mathrm{b}}$ & $83.9^{\mathrm{a}, \mathrm{b}}$ & $84.6^{\mathrm{a}, \mathrm{b}}$ & $85.1^{\mathrm{a}}$ & 0.68 & 0.027 \\
\hline \multicolumn{7}{c}{ Metabolisable Energy } \\
\hline \multicolumn{7}{c}{$\left(\mathrm{MJ} \mathrm{k9^{-1 }} \mathrm{DM}\right)$} \\
\hline Before Aeration & $10.8^{\mathrm{a}}$ & $10.4^{\mathrm{a}}$ & $10.7^{\mathrm{a}}$ & $10.7^{\mathrm{a}}$ & 0.13 & $<0.001$ \\
After Aeration & $9.6^{\mathrm{b}}$ & $10.8^{\mathrm{a}}$ & $10.7^{\mathrm{a}}$ & $10.8^{\mathrm{a}}$ & & \\
Treatment Mean & $10.2^{\mathrm{b}}$ & $10.6^{\mathrm{a}, \mathrm{b}}$ & $10.7^{\mathrm{a}}$ & $10.8^{\mathrm{a}}$ & 0.10 & 0.020
\end{tabular}

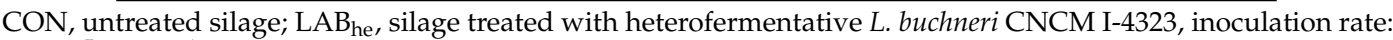
$1 \times 10^{5} \mathrm{cfu} \mathrm{g}^{-1} ; \mathrm{LAB}_{\text {heho, }}$ silage treated with a mixture of heterofermentative L. buchneri CNCM I-4323 and homofermentative $P$. acidilactici DSM 11673, total inoculation rate: $1.67 \times 10^{5} \mathrm{cfu} \mathrm{g}^{-1}$; NHS, aqueous mixture of sodium nitrite $\left(195 \mathrm{~g} \mathrm{~L}^{-1}\right)$, hexamethylene tetramine $\left(71 \mathrm{~g} \mathrm{~L}^{-1}\right)$, potassium sorbate $\left(106 \mathrm{~g} \mathrm{~L}^{-1}\right)$, applied at $2.5 \mathrm{~mL} \mathrm{~kg}$; SEM, standard error of the mean; ${ }^{a, b}$ Least-square (LS) means in rows and columns (within parameter) bearing unlike letters differ at $p<0.05$ (Tukey's test), $p$ values denote significance level of the interaction aeration $\times$ additive treatment and of the main effect of additive treatment. The main effect of aeration had $p$ values of 0.250 and 0.123 , for IVOMD and ME, respectively.

\subsection{Trial 2}

The use of the chemical additive resulted in the most efficient fermentation process as reflected by the lowest DM losses $(p<0.001)$ and highest WSC concentration $(p<0.001)$, whereas $\mathrm{LAB}_{\text {he }}$ caused an increase in DM losses and resulted in the lowest WSC content (Table 4). All treatments restricted proteolysis based on ammonia-N concentrations $(p<0.001)$. The sole use of Lactobacillus buchneri decreased lactic acid concentration $(p<0.001)$ and stimulated the production of acetic $(p<0.001)$ and propionic acids $(p<0.001)$, and n-propanol $(p<0.001)$ when compared with all other treatments. Butyric acid concentration was low in untreated silage but was further reduced by additive application $(p<0.001)$. Ethanol formation was restricted by the additives $\mathrm{LAB}_{\text {heho }}$ and NHS $(p<0.001$,) but LAB he tended to increase the content of this fermentation end-product over that of untreated silage $(p=0.051)$. The highest concentration of 1,2-propandiol was detected in treatment $\mathrm{LAB}_{\text {heho }}$ followed by NHS and $\mathrm{LAB}_{\mathrm{he}}$, and untreated silage contained only minute quantities $(p<0.001)$. All additives reduced yeast numbers $(p<0.001)$ and concurrently improved ASTA $(p<0.001)$ with a trend observed for the comparison between untreated silage and NHS application $(p=0.064)$, and between $\mathrm{LAB}_{\text {he }}$ and $\mathrm{LAB}_{\text {heho }}$ versus NHS silages $(p=0.070)$. The extent of aerobic deterioration as expressed as cumulated temperature was vastly restricted in treatments $\mathrm{LAB}_{\text {he }}$ and $\mathrm{LAB}_{\text {heho }}(p<0.001)$.

There was no additive-by-aeration interaction regarding IVOMD and ME concentration (Table 5). Across treatments, however, aeration decreased the IVOMD from 79.1 to $77.2 \%(p=0.001)$ and the ME concentration from 10.0 to $9.6 \mathrm{MJ} \mathrm{kg}^{-1} \mathrm{DM}(p<0.001)$. When IVOMD and ME values were averaged across aeration, an improvement was observed by the NHS application compared to untreated (IVOMD: 79.7 vs. $76.6 \%, p=0.006$; ME: 10.0 vs. $\left.9.5 \mathrm{MJ} \mathrm{ME} \mathrm{kg}^{-1} \mathrm{DM}, p=0.008\right)$. Trends were found for increased IVOMD of the $\mathrm{LAB}_{\text {heho }}$ treatment compared to untreated control $(p=0.076)$, and for the NHS compared to the $\mathrm{LAB}_{\text {he }}$ treatment $(p=0.086)$. 
Table 4. Fermentation characteristics, yeast count and aerobic stability of grass-clover silage in trial 2 (LSmeans and SEM in $\mathrm{g} \mathrm{kg}^{-1}$ dry matter unless stated otherwise, $n=3$ ).

\begin{tabular}{ccccccc}
\hline Parameter & CON & LAB $_{\text {he }}$ & LAB $_{\text {heho }}$ & NHS & SEM & $p$ \\
\hline DM $\left(\mathrm{g} \mathrm{kg}^{-1}\right)$ & $325^{\mathrm{b}}$ & $348^{\mathrm{a}}$ & $342^{\mathrm{a}, \mathrm{b}}$ & $359^{\mathrm{a}}$ & 5.1 & 0.009 \\
DM loss (\%) & $6.8^{\mathrm{b}}$ & $8.6^{\mathrm{a}}$ & $7.0^{\mathrm{b}}$ & $5.5^{\mathrm{c}}$ & 0.12 & $<0.001$ \\
WSC & $9.5^{\mathrm{b}}$ & $4.1^{\mathrm{c}}$ & $7.7^{\mathrm{b}, \mathrm{c}}$ & $33.9^{\mathrm{a}}$ & 0.89 & $<0.001$ \\
$\mathrm{NH}_{3}-\mathrm{N}\left(\mathrm{g} \mathrm{kg}^{-1} \mathrm{~N}\right)$ & $168^{\mathrm{a}}$ & $131^{\mathrm{b}}$ & $104^{\mathrm{c}}$ & $83^{\mathrm{d}}$ & 3.7 & $<0.001$ \\
pH & $4.75^{\mathrm{a}}$ & $4.67^{\mathrm{a}}$ & $4.40^{\mathrm{b}}$ & $4.36^{\mathrm{b}}$ & 0.018 & $<0.001$ \\
Lactic Acid & $42.5^{\mathrm{a}}$ & $28.2^{\mathrm{b}}$ & $43.6^{\mathrm{a}}$ & $45.6^{\mathrm{a}}$ & 0.96 & $<0.001$ \\
Acetic Acid & $15.9^{\mathrm{b}}$ & $32.0^{\mathrm{a}}$ & $29.0^{\mathrm{a}}$ & $19.8^{\mathrm{b}}$ & 1.40 & $<0.001$ \\
Propionic Acid & $0.2^{\mathrm{b}}$ & $1.9^{\mathrm{a}}$ & $0^{\mathrm{c}}$ & $0^{\mathrm{c}}$ & 0.04 & $<0.001$ \\
Butyric Acid & $1.5^{\mathrm{a}}$ & $0.3^{\mathrm{b}}$ & $0.3^{\mathrm{b}}$ & $0^{\mathrm{c}}$ & 0.01 & $<0.001$ \\
Ethanol & $13.7^{\mathrm{a}}$ & $18.4^{\mathrm{a}}$ & $6.6^{\mathrm{b}}$ & $3.4^{\mathrm{b}}$ & 1.05 & $<0.001$ \\
n-propanol & $0^{\mathrm{b}}$ & $2.9^{\mathrm{a}}$ & $0^{\mathrm{b}}$ & $0.3^{\mathrm{b}}$ & 0.12 & $<0.001$ \\
1,2-propanediol & $1.8^{\mathrm{d}}$ & $7.4^{\mathrm{c}}$ & $18.2^{\mathrm{a}}$ & $11.0^{\mathrm{b}}$ & 0.40 & $<0.001$ \\
Yeast Count (log cfu g $\left.{ }^{-1}\right)$ & $6.3^{\mathrm{a}}$ & $1.7^{\mathrm{c}}$ & $2.1^{\mathrm{c}}$ & $3.9^{\mathrm{b}}$ & 0.30 & $<0.001$ \\
ASTA (hours) & $37^{\mathrm{b}}$ & $>288^{\mathrm{a}}$ & $>288^{\mathrm{a}}$ & $164^{\mathrm{a}, \mathrm{b}}$ & 29.6 & $<0.001$ \\
TCUM $\left({ }^{\circ} \mathrm{C}\right)$ & $532^{\mathrm{a}}$ & $58^{\mathrm{b}}$ & $10^{\mathrm{b}}$ & $412^{\mathrm{a}}$ & 67.2 & 0.001 \\
\hline
\end{tabular}

$\mathrm{CON}$, untreated silage; $\mathrm{LAB}_{\text {he, }}$ silage treated with heterofermentative L. buchneri CNCM I-4323, inoculation rate: $1 \times 10^{5} \mathrm{cfu} \mathrm{g}^{-1} ; \mathrm{LAB}_{\text {heho, }}$, silage treated with a mixture of heterofermentative L. buchneri CNCM I-4323 and homofermentative $P$. acidilactici DSM 11673, total inoculation rate: $1.67 \times 10^{5} \mathrm{cfu} \mathrm{g}^{-1}$; NHS, aqueous mixture of sodium nitrite $\left(195 \mathrm{~g} \mathrm{~L}^{-1}\right)$, hexamethylene tetramine $\left(71 \mathrm{~g} \mathrm{~L}^{-1}\right)$, potassium sorbate $\left(106 \mathrm{~g} \mathrm{~L}^{-1}\right)$, applied at $2.0 \mathrm{~mL} \mathrm{~kg}^{-1}$; SEM, standard error of the mean; WSC, water-soluble carbohydrates; ASTA, aerobic stability, > denotes that silages were still stable upon termination of the ASTA test after $288 \mathrm{~h}$ of air exposure; TCUM, cumulated temperature during air exposure; ${ }^{a, b, c, d}$ Least-square (LS) means in rows bearing unlike letters differ at $p<0.05$ (Tukey's test).

Table 5. In vitro organic matter digestibility (IVOMD, \% of OM) and metabolisable energy ( $\mathrm{MJ} \mathrm{kg}^{-1}$ $\mathrm{DM})$ concentration of grass-clover silage in trial 2 upon silo opening and after $288 \mathrm{~h}$ of aeration as affected by silage additive use (LSmeans and SEM, $n=3$ ).

\begin{tabular}{|c|c|c|c|c|c|c|}
\hline Parameter & CON & $\mathrm{LAB}_{\text {he }}$ & $\mathrm{LAB}_{\text {heho }}$ & NHS & SEM & $p$ \\
\hline \multicolumn{7}{|c|}{ IVOMD ( $\%$ of OM) } \\
\hline Before Aeration & 77.8 & 79.0 & 79.2 & 80.3 & 0.57 & 0.786 \\
\hline After Aeration & 75.4 & 77.0 & 77.5 & 79.0 & & \\
\hline Treatment Mean & $76.6^{\mathrm{b}}$ & $78.0^{a, b}$ & $78.3^{a, b}$ & $79.7^{\mathrm{a}}$ & 0.42 & 0.006 \\
\hline \multicolumn{7}{|c|}{ Metabolisable energy } \\
\hline \multicolumn{7}{|c|}{$\left(\mathrm{MJ} \mathrm{kg}{ }^{-1} \mathrm{DM}\right)$} \\
\hline Before Aeration & 9.8 & 10.0 & 10.0 & 10.2 & 0.10 & 0.690 \\
\hline After Aeration & 9.3 & 9.6 & 9.7 & 9.9 & & \\
\hline Treatment Mean & $9.5^{b}$ & $9.8^{\mathrm{a}, \mathrm{b}}$ & $9.8^{\mathrm{a}}$ & $10.0^{\mathrm{a}}$ & 0.07 & 0.008 \\
\hline
\end{tabular}

$\mathrm{CON}$, untreated silage; $\mathrm{LAB}_{\mathrm{he}}$, silage treated with heterofermentative L. buchneri CNCM I-4323, inoculation rate: $1 \times 10^{5} \mathrm{cfu} \mathrm{g}^{-1} ; \mathrm{LAB}_{\text {heho, }}$ silage treated with a mixture of heterofermentative L. buchneri CNCM I-4323 and homofermentative P. acidilactici DSM 11673, total inoculation rate: $1.67 \times 10^{5} \mathrm{cfu} \mathrm{g}^{-1}$; NHS, aqueous mixture of sodium nitrite $\left(195 \mathrm{~g} \mathrm{~L}^{-1}\right)$, hexamethylene tetramine $\left(71 \mathrm{~g} \mathrm{~L}^{-1}\right)$, potassium sorbate $\left(106 \mathrm{~g} \mathrm{~L}^{-1}\right)$, applied at $2 \mathrm{~mL} \mathrm{~kg}^{-1}$; SEM, standard error of the mean; ${ }^{a, b}$ Least-square (LS) means in rows (within parameter) bearing unlike letters differ at $p<0.05$ (Tukey's test), $p$ values denote significance level of the interaction aeration $\times$ additive treatment and of the main effect of additive treatment. The main effect of aeration had $p$ values of 0.001 and $<0.001$ for IVOMD and ME, respectively.

\subsection{Trial 3}

All silages were well fermented with butyric acid and n-propanol not found in any of the silages (Table 6). Despite generally low DM losses during fermentation, using the additives LAB ho NHS and $\mathrm{BNP}$ resulted in a decrease by 5 to $8 \%$ compared with untreated silage $(p<0.001)$. The largest quantities of WSC were preserved by $\mathrm{LAB}_{\mathrm{ho}}$, NHS and BNP when compared with untreated silage and those inoculated with $\mathrm{LAB}_{\text {he }}$ and $\mathrm{LAB}_{\text {heho }}(p<0.001)$. Additives affected proteolysis $(p<0.001)$, $\mathrm{pH}(p<0.001)$ and lactic acid production $(p=0.007)$. The untreated silage had lower acetic acid 
concentration compared to $\mathrm{LAB}_{\text {he }}(p=0.005)$, NHS and BNP $(p<0.001)$. Regardless of composition, chemical additives restricted ethanol formation $(p<0.001)$, whereas the smallest concentrations of 1,2-propanediol were analysed in untreated silage and when $\mathrm{LAB}_{\text {ho }}$ was applied $(p<0.001)$. The highest yeast count was enumerated in $\mathrm{LAB}_{\mathrm{ho}}$-inoculated silage, whereas $\mathrm{LAB}_{\text {heho }}$, NHS and BNP treatments contained the lowest numbers $(p<0.001)$. The ASTA of the silage was improved over that, which was untreated, by all inoculants containing Lactobacillus buchneri and, even more, by chemical additives, whereas a reduction was observed by $59 \mathrm{~h}$ for $\operatorname{LAB}_{\text {ho }}(p<0.001)$. Furthermore, the extent of aerobic deterioration was lowest in silage that had received chemical additives $(p<0.001)$.

Table 6. Fermentation characteristics, yeast count and aerobic stability of grass-clover silage in trial 3 (LS means and SEM in $\mathrm{g} \mathrm{kg}^{-1}$ dry matter unless stated otherwise, $n=3$ ).

\begin{tabular}{|c|c|c|c|c|c|c|c|c|}
\hline Parameter & $\mathrm{CON}$ & $\mathbf{L A B}_{\text {ho }}$ & LAB $_{\text {he }}$ & LAB $_{\text {heho }}$ & NHS & BNP & SEM & $p$ \\
\hline $\mathrm{DM}\left(\mathrm{g} \mathrm{kg}^{-1}\right)$ & $229^{b}$ & $230^{b}$ & $231^{a, b}$ & $231^{a, b}$ & $232^{a, b}$ & $234^{a}$ & 0.8 & 0.005 \\
\hline DM loss (\%) & $6.0^{\mathrm{b}}$ & $5.6^{c}$ & $6.3^{a}$ & $6.2^{\mathrm{a}, \mathrm{b}}$ & $5.7^{c}$ & $5.5^{c}$ & 0.06 & $<0.001$ \\
\hline WSC & $10.3^{c}$ & $14.4^{\mathrm{a}, \mathrm{b}}$ & $7.8^{\mathrm{d}}$ & $8.8^{c, d}$ & $12.6^{b}$ & $16.0^{\mathrm{a}}$ & 0.41 & $<0.001$ \\
\hline $\mathrm{NH}_{3}-\mathrm{N}\left(\mathrm{g} \mathrm{kg}^{-1} \mathrm{~N}\right)$ & $73^{a, b}$ & $68^{c, d}$ & $77^{a}$ & $72^{b, c}$ & $60^{\mathrm{e}}$ & $66^{c, d}$ & 1.1 & $<0.001$ \\
\hline $\mathrm{pH}$ & $4.17^{\mathrm{a}}$ & $4.06^{b}$ & $4.18^{\mathrm{a}}$ & $4.19^{\mathrm{a}}$ & $4.19^{\mathrm{a}}$ & $4.18^{\mathrm{a}}$ & 0.014 & $<0.001$ \\
\hline Lactic Acid & $87.4^{\mathrm{a}, \mathrm{b}}$ & $95.4^{\mathrm{a}}$ & $80.5^{b}$ & $83.9^{b}$ & $81.3^{b}$ & $82.3^{b}$ & 2.35 & 0.007 \\
\hline Acetic Acid & $16.3^{\mathrm{c}, \mathrm{d}}$ & $15.1^{\mathrm{d}}$ & $20.8^{b}$ & $19.0^{b, c}$ & $23.1^{\mathrm{a}}$ & $22.0^{a, b}$ & 0.68 & $<0.001$ \\
\hline Propionic Acid & ND & ND & ND & ND & ND & ND & & \\
\hline Butyric Acid & ND & ND & ND & ND & ND & ND & & \\
\hline Ethanol & $5.2^{\mathrm{a}}$ & $4.2^{\mathrm{a}}$ & $5.3^{\mathrm{a}}$ & $4.6^{\mathrm{a}}$ & $2.6^{b}$ & $2.1^{b}$ & 0.30 & $<0.001$ \\
\hline n-propanol & $\mathrm{N}^{\mathrm{D}}$ & $\mathrm{N}^{\mathrm{D}}$ & $\mathrm{N}^{\mathrm{D}}$ & $\mathrm{N}^{\mathrm{D}}$ & $\mathrm{N}^{\mathrm{D}}$ & $\mathrm{N}^{\mathrm{D}}$ & & \\
\hline 1,2-propanediol & $2.3^{c}$ & $2.2^{\mathrm{c}}$ & $6.0^{\mathrm{a}}$ & $5.1^{\mathrm{a}, \mathrm{b}}$ & $4.9^{\mathrm{a}, \mathrm{b}}$ & $4.2^{b}$ & 0.27 & $<0.001$ \\
\hline Yeast Count $\left(\log \mathrm{cfu} \mathrm{g}^{-1}\right)$ & $3.3^{b}$ & $5.7^{\mathrm{a}}$ & $2.2^{b, c}$ & $1.7^{\mathrm{c}}$ & $1.7^{\mathrm{c}}$ & $1.8^{\mathrm{c}}$ & 0.28 & $<0.001$ \\
\hline ASTA (hours) & $164^{c}$ & $105^{d}$ & $238^{b}$ & $228^{b}$ & $>336^{a}$ & $>336^{a}$ & 8.1 & $<0.001$ \\
\hline TCUM $\left({ }^{\circ} \mathrm{C}\right)$ & $708^{a, b}$ & $811^{\mathrm{a}}$ & $452^{b}$ & $550^{a, b}$ & $27^{c}$ & $40^{c}$ & 70.2 & $<0.001$ \\
\hline
\end{tabular}

$\mathrm{CON}$, untreated silage; $\mathrm{LAB}_{\mathrm{ho}}$, silage treated with a mixture of homofermentative L. plantarum DSM 16627 and L. paracasei NCIMB 30151, total inoculation rate: $1.5 \times 10^{5} \mathrm{cfu}^{-1}$; $\mathrm{LAB}_{\mathrm{he}}$, silage treated with heterofermentative L. buchneri CNCM I-4323, inoculation rate: $1 \times 10^{5} \mathrm{cfu} \mathrm{g}^{-1} ; \mathrm{LAB}_{\text {heho, }}$, silage treated with a mixture of heterofermentative L. buchneri CNCM I-4323 and homofermentative P. acidilactici DSM 11673, total inoculation rate: $1.67 \times 10^{5} \mathrm{cfu}$ $\mathrm{g}^{-1}$; NHS, aqueous mixture of sodium nitrite $\left(195 \mathrm{~g} \mathrm{~L}^{-1}\right)$, hexamethylene tetramine $\left(71 \mathrm{~g} \mathrm{~L}^{-1}\right)$, potassium sorbate $\left(106 \mathrm{~g} \mathrm{~L}^{-1}\right)$, applied at $2 \mathrm{~mL} \mathrm{~kg}^{-1}$; BNP, aqueous mixture of sodium benzoate $\left(238 \mathrm{~g} \mathrm{~L}^{-1}\right)$, sodium nitrite $\left(119 \mathrm{~g} \mathrm{~L}^{-1}\right)$, sodium propionate $\left(12 \mathrm{~g} \mathrm{~L}^{-1}\right)$, applied at $2 \mathrm{~mL} \mathrm{~kg}^{-1}$; SEM, standard error of the mean; WSC, water-soluble carbohydrates; ND, not detected; ASTA, aerobic stability, >denotes that silages were still stable upon termination of the ASTA test after $336 \mathrm{~h}$ of air exposure; TCUM, cumulated temperature during air exposure; ${ }^{\text {a,b,c,d,e }}$ Least-square (LS) means in rows bearing unlike letters differ at $p<0.05$ (Tukey's test).

Additives had no effect on IVOMD and ME concentration in grass-clover silage before air exposure (Table 7). Silage treated with the chemical additives NHS and BNP maintained its nutritive value throughout the $336 \mathrm{~h}$ of air exposure. The use of $\mathrm{LAB}_{\text {he }}$ tended to reduce IVOMD $(p=0.092)$ and decreased $\mathrm{ME}$ content, whereas $\mathrm{CON}, \mathrm{LAB}_{\text {ho }}$ and $\mathrm{LAB}_{\text {heho }}$ showed both significantly lower IVOMD and lower ME concentration in aerated silage $(p<0.001)$. The magnitude of the decline ranged from 3.0 to 5.9 IVOMD percentage units and from 0.6 to $1.5 \mathrm{MJ} \mathrm{ME} \mathrm{kg}^{-1} \mathrm{DM}(p<0.001)$. After aeration, silage inoculated with $\mathrm{LAB}_{\text {ho }}$ had the lowest ME concentration $(p=0.040)$, and its IVOMD was significantly lower than that of $\mathrm{LAB}_{\text {he }}$, NHS and BNP $(p<0.001)$ and tended to be lower than that of the untreated silage $(p=0.071)$.

\subsection{Relationship Between the Extent of Aerobic Deterioration and the Changes in Metabolisable Energy during Aeration}

As depicted in Figure 1, an increase in cumulated temperature as an indicator for the extent of aerobic deterioration caused a larger negative change in ME concentration from silo opening until the end of air exposure after a maximum of $336 \mathrm{~h}$. Across trials, the relationship was highly significant $(p<0.001)$ and characterised by a Pearson partial correlation coefficient of -0.756 . However, differences in the magnitude of ME changes were observed among trials. 
Table 7. In vitro organic matter digestibility (IVOMD, \% of OM) and metabolisable energy ( $\mathrm{MJ} \mathrm{kg}^{-1}$ $\mathrm{DM})$ concentration of grass-clover silage in trial 3 upon silo opening and after $336 \mathrm{~h}$ of aeration as affected by silage additive (LSmeans and SEM, $n=3$ ).

\begin{tabular}{ccccccccc}
\hline Parameter & CON & LAB $_{\text {ho }}$ & LAB $_{\text {he }}$ & LAB $_{\text {heho }}$ & NHS & BNP & SEM & $p$ \\
\hline \multicolumn{7}{c}{ IVOMD $(\%$ of OM $)$} \\
\hline Before Aeration & $91.1^{\mathrm{a}, \mathrm{A}}$ & $91.6^{\mathrm{a}, \mathrm{A}}$ & $90.7^{\mathrm{a}, \mathrm{A}}$ & $91.7^{\mathrm{a}, \mathrm{A}}$ & $90.6^{\mathrm{a}, \mathrm{A}}$ & $91.5^{\mathrm{a}, \mathrm{A}}$ & 0.45 & $<0.001$ \\
After Aeration & $88.1^{\mathrm{c}, \mathrm{d}, \mathrm{B}}$ & $85.7^{\mathrm{d}, \mathrm{B}}$ & $88.4^{\mathrm{b}, \mathrm{c}, \mathrm{A}}$ & $87.4^{\mathrm{c}, \mathrm{d}, \mathrm{B}}$ & $90.3^{\mathrm{a}, \mathrm{b}, \mathrm{A}}$ & $91.7^{\mathrm{a}, \mathrm{A}}$ & \\
Treatment Mean & $89.6^{\mathrm{b}, \mathrm{c}}$ & $88.7^{\mathrm{c}}$ & $89.5^{\mathrm{b}, \mathrm{c}}$ & $89.6^{\mathrm{b}, \mathrm{c}}$ & $90.5^{\mathrm{a}, \mathrm{b}}$ & $91.6^{\mathrm{a}}$ & 0.32 & $<0.001$ \\
\hline \multicolumn{7}{c}{ Metabolisable Energy } \\
\hline \multicolumn{7}{c}{$\left(\mathrm{MJ} \mathrm{kg}{ }^{-1} \mathrm{DM}\right)$} \\
\hline Before Aeration & $11.4^{\mathrm{a}, \mathrm{A}}$ & $11.5^{\mathrm{a}, \mathrm{A}}$ & $11.3^{\mathrm{a}, \mathrm{A}}$ & $11.5^{\mathrm{a}, \mathrm{A}}$ & $11.3^{\mathrm{a}, \mathrm{A}}$ & $11.4^{\mathrm{a}, \mathrm{A}}$ & 0.07 & $<0.001$ \\
After Aeration & $10.5^{\mathrm{b}, \mathrm{B}}$ & $10.0^{\mathrm{c}, \mathrm{B}}$ & $10.7^{\mathrm{b}, \mathrm{B}}$ & $10.4^{\mathrm{b}, \mathrm{B}}$ & $11.2^{\mathrm{a}, \mathrm{A}}$ & $11.4^{\mathrm{a}, \mathrm{A}}$ & \\
Treatment Mean & $10.9^{\mathrm{b}}$ & $10.7^{\mathrm{b}}$ & $11.0^{\mathrm{b}}$ & $10.9^{\mathrm{b}}$ & $11.3^{\mathrm{a}}$ & $11.4^{\mathrm{a}}$ & 0.06 & $<0.001$ \\
\hline
\end{tabular}

$\mathrm{CON}$, untreated silage; $\mathrm{LAB}_{\mathrm{ho}}$, silage treated with a mixture of homofermentative L. plantarum DSM 16627 and L. paracasei NCIMB 30151, total inoculation rate: $1.5 \times 10^{5} \mathrm{cfu} \mathrm{g}^{-1}$; $\mathrm{LAB}_{\text {he, }}$, silage treated with heterofermentative L. buchneri $\mathrm{CNCM}$ I-4323, inoculation rate: $1 \times 10^{5} \mathrm{cfu} \mathrm{g}^{-1}$; $\mathrm{LAB}_{\text {heho }}$, silage treated with a mixture of heterofermentative L. buchneri CNCM I-4323 and homofermentative P. acidilactici DSM 11673, total inoculation rate: $1.67 \times 10^{5}$ cfu $\mathrm{g}^{-1}$; NHS, aqueous mixture of sodium nitrite $\left(195 \mathrm{~g} \mathrm{~L}^{-1}\right)$, hexamethylene tetramine $\left(71 \mathrm{~g} \mathrm{~L}^{-1}\right)$, potassium sorbate $\left(106 \mathrm{~g} \mathrm{~L}^{-1}\right)$, applied at $2 \mathrm{~mL} \mathrm{~kg}^{-1}$; BNP, aqueous mixture of sodium benzoate $\left(238 \mathrm{~g} \mathrm{~L}^{-1}\right)$, sodium nitrite $\left(119 \mathrm{~g} \mathrm{~L}^{-1}\right)$, sodium propionate $\left(12 \mathrm{~g} \mathrm{~L}^{-1}\right)$, applied at $2 \mathrm{~mL} \mathrm{~kg}^{-1}$; SEM, standard error of the mean; Least-square (LS) means within parameter bearing unlike lower case letters in rows ${ }^{\mathrm{a}, \mathrm{b}, \mathrm{c}, \mathrm{d}}$ and unlike upper case letters in columns ${ }^{\mathrm{A}, \mathrm{B}}$ differ at $p<0.05$ (Tukey's test); $p$ values denote significance level of the interaction aeration $\times$ additive treatment and of the main effect of additive treatment. The main effect of aeration had $p$ values of $<0.001$ and $<0.001$ for IVOMD and $\mathrm{ME}$, respectively.

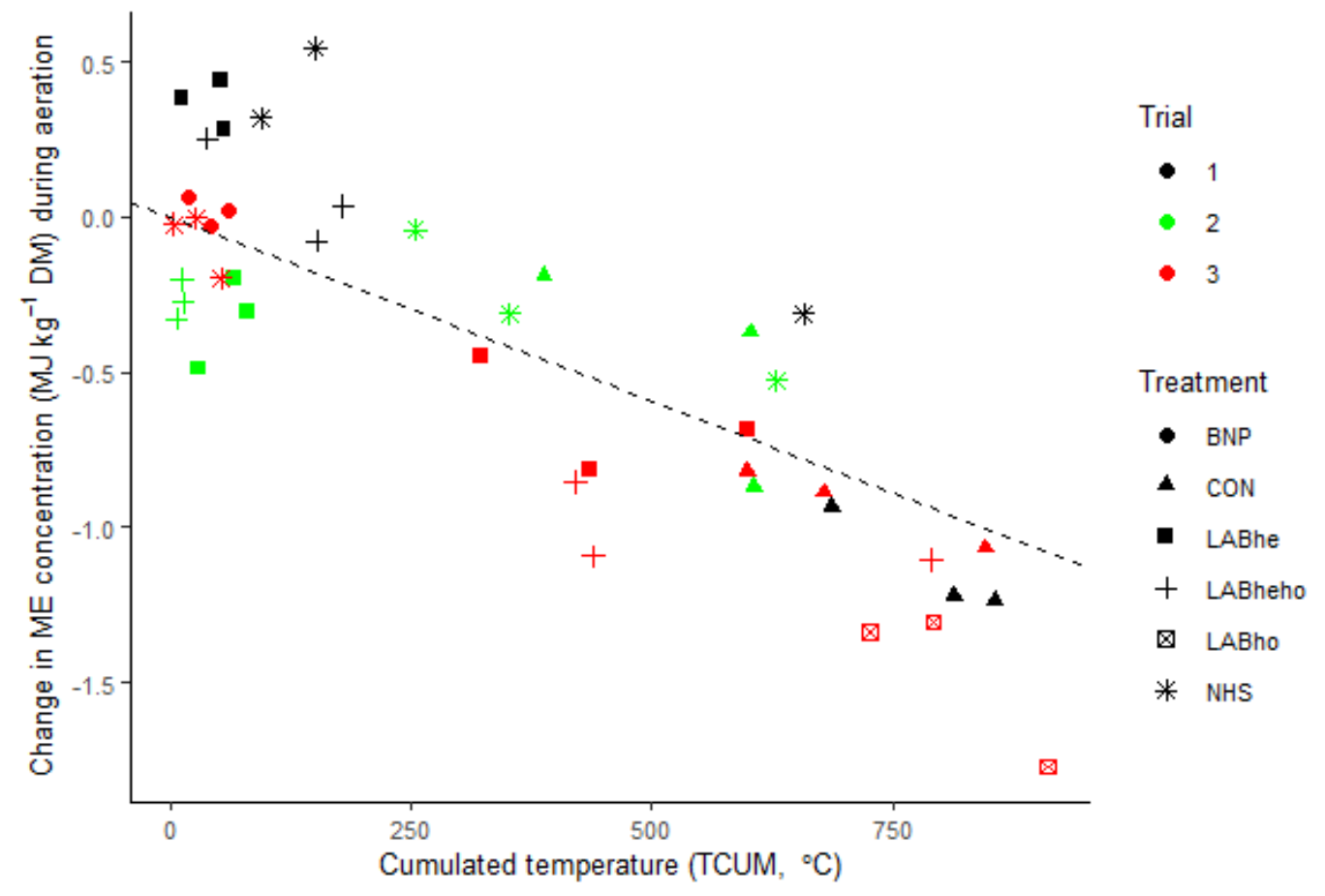

Figure 1. Relationship between aerobic deterioration expressed as cumulated temperature (TCUM, ${ }^{\circ} \mathrm{C}$ ) and the change in ME concentration $\left(\mathrm{MJ} \mathrm{kg}^{-1} \mathrm{DM}\right)$ during aeration of grass and grass-clover silage across trials and additive treatments; $\mathrm{CON}$, untreated silage; $\mathrm{LAB}_{\mathrm{ho}}$, silage treated with a mixture of homofermentative L. plantarum DSM 16627 and L. paracasei NCIMB 30151, total inoculation rate: $1.5 \times 10^{5} \mathrm{cfu} \mathrm{g}^{-1} ; \mathrm{LAB}_{\mathrm{he}}$, silage treated with heterofermentative L. buchneri CNCM I-4323, inoculation 
rate: $1 \times 10^{5} \mathrm{cfu} \mathrm{g}^{-1}$; $\mathrm{LAB}_{\text {heho, }}$, silage treated with a mixture of heterofermentative L. buchneri $\mathrm{CNCM}$ I-4323 and homofermentative P. acidilactici DSM 11673, total inoculation rate: $1.67 \times 10^{5} \mathrm{cfu} \mathrm{g}^{-1}$; NHS, aqueous mixture of sodium nitrite $\left(195 \mathrm{~g} \mathrm{~L}^{-1}\right)$, hexamethylene tetramine $\left(71 \mathrm{~g} \mathrm{~L}^{-1}\right)$, potassium sorbate (106 $\mathrm{g} \mathrm{L}^{-1}$ ), applied at $2.5 \mathrm{~mL} \mathrm{~kg}^{-1}$ (trial 1) or at $2 \mathrm{~mL} \mathrm{~kg}^{-1}$ (trials 2 and 3); BNP, aqueous mixture of sodium benzoate $\left(238 \mathrm{~g} \mathrm{~L}^{-1}\right)$, sodium nitrite $\left(119 \mathrm{~g} \mathrm{~L}^{-1}\right)$, sodium propionate $\left(12 \mathrm{~g} \mathrm{~L}^{-1}\right)$, applied at $2 \mathrm{~mL} \mathrm{~kg}^{-1} ; n=42$, Pearson partial correlation coefficient $=-0.756, p<0.001$, estimate of common slope $=-0.0015$, Root mean square error $=0.298$.

\section{Discussion}

\subsection{Effects of Additives on Fermentation Characteristics, Yeast Count and Aerobic Stability}

In general, DM losses during fermentation, which are a suitable indicator for the efficiency of the fermentation process, were low in all trials. Regardless of additive use, our observations support information by Borreani et al. [1] on potential DM losses caused by in-silo respiration and fermentation under good silo management conditions on commercial farms. The observed reduction in $\mathrm{DM}$ loss by chemical additives in all trials and by $\mathrm{LAB}_{\mathrm{ho}}$ tested in trial 3 only highlight their role in inhibiting fermentation pathways by undesired microorganisms, leading to more efficient fermentation accompanied by protection of WSC from degradation. On the contrary, inoculation with solely Lactobacillus buchneri increased DM losses and reduced WSC concentration. This substantiates conclusions drawn from a meta-analysis on grass and small-grain silage by Kleinschmit and Kung [19] who reported decreased DM recovery by 1.55 percentage units of silages inoculated with Lactobacillus buchneri. Anaerobic degradation of lactic acid by this species to form acetic acid and 1,2-propandiol, and ethanol production, ultimately leads to $\mathrm{CO}_{2}$ production, and DM losses [20,42].

According to Kung et al. [43], all experimental silages were of typical and good quality. Statistically significant differences frequently detected among treatments in all trials for numerous silage characteristics were of limited, or no, biological relevance, but some indices warrant discussion. Our findings confirm the unique mode of action of Lactobacillus buchneri resulting in lower lactic acid concentrations, larger acetic acid and 1,2-propanediol contents and higher silage $\mathrm{pH}$ as reported by Kleinshmit and Kung [19] for grass and small grain silage, and by Schmidt et al. [44] for lucerne silage. The combined application with the homofermentative Pediococcus pentosaceus, partially alleviated the influence of Lactobacillus buchneri alone on fermentation and DM losses in our experiments and in previous studies $[20,21,44]$, which is the rationale behind using dual-purpose inoculants in grass and legume silages. However, the magnitude of the effect is very likely to depend on the strains used [45], the total inoculation rate and the ratio between hetero- and homofermentative species. The metabolic end-products of 1,2-propanediol utilisation by Lactobacillus diolivorans-propionic acid and n-propanol [46] - were only detected in trial 2 at minute concentrations. The most plausible explanation for our findings may be that this species was not present in the epiphytic bacterial flora at ensiling, it was not metabolically active during the fermentation process, or it was outcompeted by other microorganisms. Moreover, Gomes et al. [47] observed an effect of DM at ensiling of oat silage and detected higher concentrations of n-propanol and propionic acid in silage of $21 \%$ DM compared to silage of 30\% DM. Therefore, it seems likely that wild, epiphytic Lactobacillus diolivorans strains possess only limited osmotolerance.

The tested chemicals consistently restricted proteolysis in all trials confirming previous observations for mixtures containing antibacterial and antimycotic substances $[24,48]$ to suppress certain microbial populations, e.g., clostridia and enterobacteria, which are known ammonia-producers [5]. The strong inhibitory effect can be ascribed mainly to the antibacterial activity of sodium nitrite and hexamethylene tetramine [24,48]. Our data support earlier reports on highly varying levels of proteolytic activity by Lactobacillus buchneri [20]. Although we consistently found lower ammonia-N concentrations by combining Lactobacillus buchneri with $\mathrm{LAB}_{\mathrm{ho}}$, this strategy has not always proven to be successful $[20,44]$. Apparently, other factors, including forage type, composition of the epiphytic 
microflora [20], or the strains of $\mathrm{LAB}_{\text {ho }}$ used to formulate the dual-purpose inoculant, play a role [45], making it difficult to predict the magnitude of the inoculation effect.

In our trials, the most relevant and significant effects of additive application were detected on yeast numbers and ASTA. Obviously, the high yeast counts in excess of the threshold value of $\log _{10} 5.0 \mathrm{cfu}^{-1}$, which is indicative for rapid aerobic deterioration [49] caused untreated silage to spoil within less than 2 days whereas, in trial 3, lower yeast numbers ensured longer ASTA, as was shown previously by Auerbach et al. [21]. Additives composed of Lactobacillus buchneri alone or in combination with $\mathrm{LAB}_{\text {ho }}$ consistently improved ASTA over that of untreated silage due to lower yeast counts substantiating previous observations from a range of silage types $[14,19,20]$. However, this cannot be explained by higher acetic acid concentrations $[19,50]$ in trials 1 and 3 as the acetic acid concentrations of $\mathrm{LAB}_{\text {heho }}$-treated silages were not different from those of untreated silage. Apparently, other unknown factors may have played a role. Our findings that treatment with chemical additives outperformed those inoculated with $\mathrm{LAB}_{\text {he }}$ and $\mathrm{LAB}_{\text {heho }}$ in trial 3 confirmed previous observations in maize silage, which had received treatment with an additive containing strong antimycotic ingredients, e.g., sodium benzoate and potassium sorbate [27]. This can be attributed to the much stronger effect of these ingredients on yeasts compared to acetic acid [51,52], which is produced by Lactobacillus buchneri. Application of too low rates of the chemical NHS may have caused the additive not to be able to show its full potential to enhance ASTA under the specific conditions in trials 1 and 2, as dosage was shown to be crucial regarding the magnitude of effect $[15,25,27]$. Even more so, the variable effect of the chemical NHS can likely be ascribed to inhomogeneous additive application and distribution as one replicate silage had very low ASTA (trial 1: $50 \mathrm{~h}$; trial 2: $52 \mathrm{~h}$ ), whereas the remaining two replicates were more stable, or did not heat-up throughout the entire period of air exposure. In line with results from other studies [13-15,21], the use of $\mathrm{LAB}_{\text {ho }}$ substantially increased the risk of aerobic instability due to a shift in fermentation pattern towards more lactic acid, which has no inhibitory effect on yeasts and moulds [51] and can be utilised by a range of fungi as carbon and energy source $[49,53]$. Thus, the use of inoculants solely composed of $\mathrm{LAB}_{\text {ho }}$ is discouraged to grass-dominated leys under farm conditions when silage feed-out rate is low, resulting in long exposure times to air.

\subsection{Effects of Aeration and Additives on Silage Nutritive Value}

The lack of an additive effect on IVOMD and ME in silage prior to exposure to air is consistent with observations by Oliveira et al. [13] who employed a meta-analysis to evaluate the effects of inoculation with homofermentative and facultatively heterofermentative LAB on DM digestibility (DMD) in a range of silage types, and by Addah et al. [54] on maize and whole-crop wheat silage. Earlier reports revealed that DMD was improved in only $30 \%$ of the studies, but when an improvement was found the effect was as high as 5 percentage units and paralleled the improvement in DM recovery [17]. On the contrary, an increase in DMD by treating grass with $\mathrm{LAB}_{\text {ho }}$ alone or in combination with sodium benzoate improved in vivo organic matter digestibility (OMD) by 4.8 percentage units mainly due to large improvements in fermentation quality by decreasing butyric acid concentrations from $21 \mathrm{~g} \mathrm{~kg}^{-1} \mathrm{DM}$ in untreated silage to $6 \mathrm{~g} \mathrm{~kg}^{-1} \mathrm{DM}$ in silage treated with $\mathrm{LAB}_{\mathrm{ho}}$ alone and to $2 \mathrm{~g} \mathrm{~kg}^{-1}$ $\mathrm{DM}$ in silage that had received $\mathrm{LAB}_{\mathrm{ho}}$ in combination with sodium benzoate [16]. As highlighted by Brüsemeister et al. [55,56], the effect of inoculation on OMD of grass and maize silage with a dual-purpose product strongly depended on other factors, including DM, crude fibre content, variety, and even the laboratory performing the analyses, thereby explaining the large variability of additive effects on silage digestibility between studies.

We showed in all trials that aeration detrimentally affected the nutritive value of grass and grass-clover silage, but the magnitude of the effect varied largely. Considering untreated silage in trials 1 and 3 only, IVOMD declined by 6.9 percentage units and 3.0 percentage units, and ME content decreased by $1.2 \mathrm{MJ} \mathrm{kg}^{-1} \mathrm{DM}$ and $0.9 \mathrm{MJ} \mathrm{kg}^{-1} \mathrm{DM}$, respectively. Those nutritive losses need to be compensated for in diet formulations by increased concentrate supplementation, thereby increasing feed costs for the farmer. This assumption is supported by Chen et al. [28], who reported losses in 
DMD only after significant amounts of $\mathrm{CO}_{2}$, being another indicator for aerobic microbial activity, had been produced in whole-crop wheat silage. Our results on changes in nutritive value showed that losses in IVOMD per day of heating were somewhat larger in trial 1 ( 0.71 percentage units) than in trials 2 ( 0.23 percentage units) and 3 ( 0.42 percentage units). These values were generally much lower than that found by Nadeau et al. [34], reporting an IVOMD decline during aeration of maize silage by 1.4 percentage units per day of heating. The variation observed in our trials compared to the published literature can be associated to differences in silage type, extent of aerobic deterioration, and quantity and type of available substrates, which can be utilised by spoilage microorganisms, as highlighted by Pitt et al. [57]. Although some spoilage yeasts can metabolise starch [58], WSC and lactic acid constitute the principal carbon sources for fungal growth in grass silage even though growth rates are higher on WSC compared with lactic acid as substrate [57]. The discrepancy in the magnitude of losses in nutritive value found in our trials and other studies may be ascribed to a varying intensity of aerobic deterioration processes, as reflected by TCUM, as the highest TCUM of $786^{\circ} \mathrm{C}$ in trial 1 was accompanied by the highest losses in IVOMD during aeration of untreated silage. Apparently, higher WSC concentrations (74.1 $\mathrm{g} \mathrm{kg}^{-1} \mathrm{DM}$ in trial 1) resulted in a larger extent of aerobic deterioration than found in trials 2 (WSC: $9.5 \mathrm{~g} \mathrm{~kg}^{-1} \mathrm{DM}$ ) and 3 (WSC: $10.3 \mathrm{~g} \mathrm{~kg}^{-1} \mathrm{DM}$ ), or by Auerbach and Theobald [59] in early-cut whole-crop rye (TCUM: $285^{\circ} \mathrm{C}$, WSC: $15.2 \mathrm{~g} \mathrm{~kg}^{-1} \mathrm{DM}$ ). Our data provided strong evidence that the total concentration of WSC and lactic acid in silage at silo opening has a significant impact on the extent of aerobic deterioration when data from our trials (excluding silage treated with chemical additives) were taken into consideration $\left(\mathrm{R}^{2}=0.67 ; p<0.001, n=30\right.$, Figure $\mathrm{S} 1$ in supplementary material). However, other factors affecting the rate of decline in nutritive value during aeration may also play a role, including the total length of air exposure and the length of time at which silage temperature remains at least $2{ }^{\circ} \mathrm{C}$ above ambient, and the interval of temperature recordings [30]. It is also worthwhile mentioning here that the response in digestibility changes to aerobic deterioration is usually delayed compared with the development of yeasts and aerobic stability. Chen and Weinberg [28] clearly demonstrated that silage made from whole-crop wheat harvested at milk stage of maturity spoiled rapidly within 2 days of air exposure as reflected by an increase in $\mathrm{CO}_{2}$ production and yeast count in excess of $\log _{10} 7.0 \mathrm{cfu} \mathrm{g}^{-1}$, but decreased WSC concentration and DMD were not detected before day 4 of air exposure. Despite close linear relationships between ASTA and TCUM (Figure S2, in supplementary material) in our trials (trial 1: $\mathrm{R}^{2}=0.96, p<0.001, n=12$; trial 2: $\mathrm{R}^{2}=0.89, p<0.001, n=12$; trial 3: $\left.\mathrm{R}^{2}=0.92, p<0.001, n=18\right)$ and by Auerbach and Theobald [59], $\mathrm{R}^{2}=0.94, p<0.001, n=15$, TCUM may be a more suitable attribute compared to ASTA to evaluate the potential effects of aeration on the nutritive value as, occasionally, silages become aerobically unstable rapidly after silo opening, but the extent and the duration of the temperature increase remain at a low level $[26,60,61]$.

Additives that improved aerobic stability and decreased TCUM in our trials also potentially alleviated the impact of air on nutritive value of grass silage. Clear-cut effects in trial 1 for Lactobacillus buchneri-containing inoculants and the chemical additive and in trial 3 for the two tested chemicals confirmed previous results on maize silage [29], showing that no losses in nutritive value occur when silage remained stable throughout the entire period of aeration. Whenever additive treatment only delays the onset of aerobic instability and there is sufficient time for spoilage microorganisms to grow until the termination of the ASTA test, as seen in trial 3 for treatments $\mathrm{LAB}_{\text {he }}(98 \mathrm{~h}$ from onset of instability to termination of aeration) and $\mathrm{LAB}_{\text {heho }}(108 \mathrm{~h}$ from onset of instability to termination of aeration), then losses in nutritive value may occur. This substantiates findings by Tabacco et al. [30] on maize and sorghum silage who observed that, despite significant improvements in ASTA by LAB ${ }_{\text {he }}$ inoculation, milk production potential declined during aeration for $336 \mathrm{~h}$, but that this decline started at a later stage and was less pronounced than in untreated and $\mathrm{LAB}_{\mathrm{ho}}$-inoculated silage. The likely reason why the chemical NHS maintained the nutritive value of grass silage from silo opening throughout subsequent aeration in trial 1 may be that only one of the replicate silages of this treatment heated up, making it impossible to detect an effect on IVOMD and ME. Evaluating data from before and after 
aeration separately, the lack of an additive effect in trial 2 on IVOMD and ME content was unexpected and cannot be explained as there were large differences between treatments in terms of aerobic stability with treatments $\mathrm{LAB}_{\text {he }}$ and $\mathrm{LAB}_{\text {heho }}$ showing no signs of heating at all. Thus, further studies are warranted to elucidate other factors that may have an impact on changes in nutritive value.

\section{Conclusions}

Silage additives showed expected effects according to their different modes of action. Untreated silage had high yeast counts, resulting in rapid spoilage upon exposure to air. The detrimental effects of air could be minimised, or completely prevented, by chemical additives or L. buchneri-containing inoculants that have the potential to improve aerobic stability. Consequently, the use of these additives in grass silage is recommended to maintain the nutritive value from silo opening during feed-out. However, further studies are needed and encouraged to broaden the knowledge on changes in nutritive value of grass and grass-clover silage as affected by aeration and silage additive use.

Supplementary Materials: The following are available online at http://www.mdpi.com/2073-4395/10/9/1229/s1.

Author Contributions: Conceptualisation, H.A. and E.N.; methodology, H.A. and E.N.; software, E.N.; validation E.N.; formal analysis, E.N.; investigation, H.A.; resources, H.A. and E.N.; data curation, H.A. and E.N.; writing-original draft preparation, H.A.; writing-review and editing, H.A. and E.N.; visualisation, H.A. and E.N.; supervision, E.N.; project administration, E.N.; funding acquisition, H.A. and E.N. All authors have read and agreed to the published version of the manuscript.

Funding: This research was primarily funded by Region Västra Götaland, Skara, Sweden, the Swedish University of Agricultural Sciences (SLU), The Rural Economy and Agricultural Society Sjuhärad, Sweden, and the International Silage Consultancy (ISC), Germany, with minor additional funding by the KONSIL Europe GmbH, Germany.

Acknowledgments: We greatly acknowledge the support by Kirsten Weiss, Humboldt University, Berlin, Germany, for running fermentation pattern analyses and the laboratory of the Department of Animal Nutrition and Management, Swedish University of Agricultural Sciences (SLU), Uppsala, Sweden for determining silage in vitro organic matter digestibility. We are also deeply indebted to Jan-Eric Englund, Department of Biosystems and Technology, Alnarp, Sweden, for statistical advice.

Conflicts of Interest: Horst Auerbach is a shareholder of KONSIL Europe GmbH.

\section{References}

1. Borreani, G.; Tabacco, E.; Schmidt, R.J.; Holmes, B.J.; Muck, R.E. Silage review: Factor affecting dry matter and qualitative losses in silages. J. Dairy Sci. 2018, 101, 3952-3979. [CrossRef] [PubMed]

2. Tabacco, E.; Comino, L.; Borreani, G. Production efficiency, costs and environmental impacts of conventional and dynamic forage systems for dairy farms in Italy. Europ. J. Agron. 2018, 99, 1-12. [CrossRef]

3. Åby, B.A.; Randby, A.T.; Bonesmo, H.; Aass, L. Impact of grass silage quality on greenhouse gas emissions from dairy and beef production. Grass Forage Sci. 2019, 74, 525-534. [CrossRef]

4. Ranck, E.J.; Holden, L.A.; Dillon, J.A.; Rotz, C.A.; Soder, K.J. Economic and environmental effects of double cropping winter annuals and corn using the Integrated Farm System Model. J. Dairy Sci. 2020, 103, 3804-3815. [CrossRef] [PubMed]

5. Rooke, J.A.; Hatfield, R.D. Biochemistry of ensiling. In Silage Science and Technology; Buxton, D.R., Muck, R.E., Holmes, H.J., Eds.; American Society of Agronomy, Inc.: Madison, WI, USA, 2003; pp. 95-139.

6. Kung, L., Jr.; Sheperd, A.C.; Smagala, A.M.; Endres, K.M.; Bessett, C.A.; Ranjit, N.K.; Glancey, J.L. The effects of preservatives based on propionic acid on the fermentation aerobic stability of corn silage and a total mixed ration. J. Dairy Sci. 1998, 81, 1322-1330. [CrossRef]

7. Salvo, P.A.R.; Schonell, E.P.; Daniel, J.L.P.; Santos, M.C.; Morais, G.; Winckler, J.P.; Silva, J.; Nussio, L.G. Effects of Pichia norvegensis and air exposure on the nutritive value of corn silages for dairy cows. In Proceedings of the XVIIth International Silage Conference, Piracicaba, Brazil, 1-3 July 2015; pp. 70-71.

8. Windle, M.; Kung, L., Jr. The effect of a feed additive on the feeding value of a silage based TMR exposed to air. J. Dairy Sci. 2013, 96, 16.

9. Fink-Gremmels, J. Mycotoxins in cattle feeds and carry-over to dairy milk: A review. Food Add. Contam. 2008, 25, 172-180. [CrossRef] 
10. Ogunade, I.M.; Martinez-Tuppia, C.; Queiroz, O.C.M.; Jiang, Y.; Drouin, P.; Wu, F.; Vyas, D.; Adesogan, A.T. Silage review: Mycotoxins in silage: Occurrence, effects, prevention, mitigation. J. Dairy Sci. 2018, 101, 4034-4059. [CrossRef]

11. Kung, L., Jr.; Stokes, M.R.; Lin, C.J. Silage additives. In Silage Science and Technology; Buxton, D.R., Muck, R.E., Holmes, H.J., Eds.; American Society of Agronomy, Inc. Publishers: Madison, WI, USA, 2003; pp. 305-360.

12. Muck, R.E.; Nadeau, E.M.G.; McAllister, T.A.; Contreras-Govea, F.E.; Santos, M.C.; Kung, L., Jr. Silage review: Recent advances and future uses of silage additives. J. Dairy Sci. 2018, 101, 3980-4000. [CrossRef]

13. Oliveira, A.S.; Weinberg, Z.G.; Ogunade, I.M.; Cervantes, A.A.P.; Arriola, K.G.; Jiang, Y.; Kim, D.; Li, X.; Gonçalves, M.C.M.; Vyas, D.; et al. Meta-analysis of effects of inoculation with homofermentative and facultative heterofermentative lactic acid bacteria on silage fermentation, aerobic stability, and the performance of dairy cows. J. Dairy Sci. 2017, 100,4587-4603. [CrossRef]

14. Honig, H.; Thaysen, J. 10 years testing of silage additives by dlg-A comprehensive data evaluation. In Proceedings of the XIIIth International Silage Conference, Auchincruive, Scotland, 11-13 September 2002.

15. Auerbach, H.; Nadeau, E. Chemical additives for silages: When to use it and what are the options? In Proceedings of the VIth International Symposium on Forage Quality and Conservation, Piracicaba, Brazil, 7-8 November 2019.

16. Honig, H.; Schild, G.-J.; Weissbach, F.; Daenicke, R. Effect of a combination of lactic acid bacteria with formate and benzoate as a silage additive for grass under farm conditions. In Proceedings of the XIth International Silage Conference., Aberystwyth, UK, 8-11 September 1996.

17. Muck, R.E.; Kung, L., Jr. Effects of silage additives on ensiling. In Proceedings of the Silage: Field to Feedbunk, North American Conference, Hershey, PA, USA, 11-13 February 1997.

18. Bernardi, A.; Härter, C.J.; Silva, A.W.L.; Reis, R.A.; Rabelo, C.H.S. A meta-analysis examining lactic acid bacteria inoculants for maize silage: Effects on fermentation, aerobic stability, nutritive value and livestock production. Grass Forage Sci. 2019, 74, 596-612. [CrossRef]

19. Kleinschmit, D.H.; Kung, L., Jr. A meta-analysis of the effects of Lactobacillus buchneri on the fermentation and aerobic stability of corn and grass and small-grain silages. J. Dairy Sci. 2006, 89, 4005-4013. [CrossRef]

20. Driehuis, F.; Oude Elferink, S.J.W.H.; van Wikselaar, P.G. Fermentation characteristics and aerobic stability of grass silage inoculated with Lactobacillus buchneri, with or without homofermentative lactic acid bacteria. Grass Forage Sci. 2001, 56, 330-343. [CrossRef]

21. Auerbach, H.; Weiss, K.; Theobald, P.; Nadeau, E. Effects of inoculant type on dry matter losses, fermentation pattern, yeast count and aerobic stability of green rye silages. In Proceedings of the 12. BOKU-Symposium Tierernährung, Vienna, Austria, 11 April 2013.

22. Haigh, P.M.; Parker, J.W.G. Effect of silage additives and wilting on silage fermentation, digestibility and intake, and on live weight change in young cattle. Grass Forage Sci. 1985, 40, 429-436. [CrossRef]

23. Steen, R.W.J. Recent advances in the use of silage additives for dairy cattle. In Proceedings of the Occasional Symposium No. 25-Management issues for the grassland farmer in the 1990's, Malvern, UK, 26-27 November 1990.

24. Auerbach, H.; Nadeau, E.; Weiss, K.; Theobald, P. 2016a. Effects of sodium nitrite-containing additives on dry matter losses, fermentation pattern and biogenic amine formation in lucerne and cocksfoot silage. In Proceedings of the 17th International Conference on Forage Conservation, Horny Smokovec, Slovak Republic, 27-29 September 2016.

25. Auerbach, H.; Nadeau, E. Effects of chemical additives on whole-crop maize silage traits. In Proceedings of the 22nd International Grassland Congress, Sydney, NSW, Australia, 15-19 September 2013.

26. Bernardes, T.F.; de Oliveira, I.L.; Lara, M.A.S.; Casagrande, D.R.; Avila, C.L.S.; Pereira, O.G. Effects of potassium sorbate and sodium benzoate at two application rates on fermentation and aerobic stability of maize silage. Grass Forage Sci. 2014, 70, 491-498. [CrossRef]

27. Kung, L., Jr.; Smith, M.L.; da Silva, E.B.; Windle, M.C.; da Silva, T.C.; Polukis, S.A. An evaluation of the effectiveness of a chemical additive based on sodium benzoate, potassium sorbate, and sodium nitrite on the fermentation and aerobic stability of corn silage. J. Dairy Sci. 2018, 101, 5949-5960. [CrossRef]

28. Chen, Y.; Weinberg, Z. Changes during aerobic exposure of wheat silages. Anim. Feed Sci. Technol. 2009, 154, 76-82. [CrossRef] 
29. Nadeau, E.; Svensson, E.; Zaralis, K.; Helander, C.; Pauly, T.; Arnesson, A. Effects of additive on aerobic stability and nutritive value of maize silage stored during different time periods when harvested at advancing maturity stages. In Proceedings of the Advances in Animal Biosciences-8th International Symposium on the nutrition of herbivores, Aberythwyth, UK, 6-9 September 2011.

30. Tabacco, E.; Righi, F.; Quarantelli, A.; Borreani, G. Dry matter and nutritional losses during aerobic deterioration of corn and sorghum silages as influenced by different lactic acid bacteria inocula. J. Dairy Sci. 2011, 94, 1409-1419. [CrossRef]

31. Weissbach, F.; Strubelt, C. Correcting the dry matter content of grass silages as a substrate for biogas production. J. Agric. Eng. 2008, 63, 210.

32. Weissbach, F. A simple method for the correction of fermentation losses measured in laboratory silos. In Proceedings of the XIVth International Silage Conference, Belfast, UK, July 2005.

33. Van Soest, P.J.; Robertson, J.B.; Lewis, B.A. Methods for dietary fiber, neutral detergent fiber, and nonstarch polysaccharides in relation to animal nutrition. J. Dairy Sci. 1991, 74, 3583-3597. [CrossRef]

34. Weiss, K.; Kaiser, E. Milchsäurebstimmung in Silageextrakten mit Hilfe der HPLC [Lactic acid determination in silage extracts by HPLC]. Das Wirtschaftseigene Futter 1995, 41, 69-80.

35. Weiss, K.; Kroschewski, B.; Auerbach, H. Effects of air exposure, temperature and additives on fermentation characteristics, yeast count, aerobic stability and volatile organic compounds in corn silage. J. Dairy Sci. 2016, 99, 8053-8069. [CrossRef] [PubMed]

36. Von Lengerken, J.; Zimmermann, K. Handbuch Futtermittelprüfung [Handbook Feed Evaluation], 1st ed.; Deutscher Landwirtschaftsverlag: Berlin, Germany, 1991; pp. 206-267. (In German)

37. ISO 21527-1. Microbiology of Food and Animal Feeding Stuffs-Horizontal Method for the Enumeration of Yeasts and Moulds_Part 1: Colony Count Technique in Products with Water Activity Greater than 0.95; International Organization for Standardization: Geneva, Switzerland, 2008.

38. Honig, H. Evaluation of aerobic stability. In Proceedings of the EUROBAC Conference, Uppsala, Sweden, 12-16 August 1986.

39. Lindgren, E. Vallfodrets Näringsvärde Bestämt In Vivo Och Med Olika Laboratoriemetoder; Report No. 45; Department of Animal Nutrition and Management, Swedish University of Agricultural Sciences: Uppsala, Sweden, 1979. (In Swedish)

40. Lindgren, E. Nykalibrering av VOS Metoden för Bestämning av Energivärde Hos Vallfoder; Working Paper; Department of Animal Nutrition and Management, Swedish University of Agricultural Sciences: Uppsala, Sweden, 1983. (In Swedish)

41. Lindgren, E. Fodrets Energivärde; Course Paper Feed Science HNU 3; Department of Animal Nutrition and Management, Swedish University of Agricultural Sciences: Uppsala, Sweden, 1988. (In Swedish)

42. Oude-Elferink, S.J.W.H.; Krooneman, J.; Gotschal, J.C.; Spoelstra, S.F.; Faber, F.; Driehuis, F. Anaerobic degradation of lactic acid to acetic acid and 1,2-propandediol by Lactobacillus buchneri. Appl. Environm. Microbiol. 2001, 67, 125-132. [CrossRef] [PubMed]

43. Kung, L., Jr.; Shaver, R.D.; Grant, R.J.; Schmidt, R.J. Silage review: Interpretation of chemical, microbial and organoleptic components of silages. J. Dairy Sci. 2018, 101, 4020-4033. [CrossRef] [PubMed]

44. Schmidt, R.J.; Hu, W.; Mills, J.A.; Kung, L., Jr. The development of lactic acid bacteria and Lactobacillus buchneri and their effects on the fermentation of alfalfa silage. J. Dairy Sci. 2009, 92, 5005-5010. [CrossRef] [PubMed]

45. Reich, L.J.; Kung, L., Jr. Effects of combining Lactobacillus buchneri 40788 with various lactic acid bacteria on the fermentation and aerobic stability of corn silage. Anim. Feed Sci. Technol. 2010, 159, 105-109. [CrossRef]

46. Krooneman, J.; Faber, F.; Alderkamp, A.C.; Oude Elferink, S.J.H.W.; Driehuis, F.; Cleenwerck, I.; Swings, J.; Gottschal, J.C.; Vancanneyt, M. Lactobacillus diolivorans sp. nov., a 1,2-propanediol-degrading bacterium isolated from aerobically stable maize silage. Int. J. Syst. Evol. Microbiol. 2002, 52, 639-646. [CrossRef]

47. Gomes, A.L.M.; Jacovaci, F.A.; Bolsson, D.C.; Nussio, L.G.; Jobim, C.C.; Daniel, J.L.P. Effect of light wilting and heterolactic inoculant on the formation of volatile organic compounds, fermentative losses and aerobic stability of oat silage. Anim. Feed Sci. Technol. 2019, 247, 194-198. [CrossRef]

48. Lingvall, P.; Lättemäe, P. Influence of hexamine and sodium nitrite in combination with sodium benzoate and sodium propionate on fermentation and hygienic quality of wilted and long cut grass silage. J. Sci. Food Agric. 1999, 79, 257-264. [CrossRef] 
49. Jonsson, A.; Pahlow, G. Systematic classification and biochemical characterization of yeasts growing in grass silage inoculated with Lactobacillus cultures. Anim. Res. Dev. 1984, 20, 7-22.

50. Schmidt, R.J.; Kung, L., Jr. The effects of Lactobacillus buchneri with or without a homolactic bacterium on the fermentation and aerobic stability of corn silages made at different locations. J. Dairy Sci. 2010, 93, 1616-1624. [CrossRef] [PubMed]

51. Woolford, M.K. Microbiological screening of the straight chain fatty acids $\left(C_{1}-C_{12}\right)$ as potential silage additives. J. Sci. Food Agric. 1975, 26, 219-228. [CrossRef] [PubMed]

52. Woolford, M.K. Microbiological screening of food preservatives, cold sterilants and specific antimicrobial agents as silage additives. J. Sci. Food Agric. 1975, 26, 229-237. [CrossRef]

53. Santos, M.C.; Golt, C.; Joerger, R.D.; Mechor, G.D.; Mourão, G.B.; Kung, L., Jr. Identification of the major yeasts isolated from high moisture corn and corn silages in the United States using genetic and biochemical methods. J. Dairy Sci. 2016, 100, 1151-1160. [CrossRef] [PubMed]

54. Addah, W.; Baah, J.; Groenewegen, P.; Okine, E.K.; McAllister, T.A. Comparison of the fermentation characteristics, aerobic stability and nutritive value of barley and corn silages ensiled with or without a mixed bacterial inoculant. Can. J. Anim. Sci. 2011, 91, 133-146. [CrossRef]

55. Brüsemeister, F.; Kalzendorf, C.; Losand, B.; Ruser, B.; Thaysen, J. Influence of treating grass with a ferulate esterase releasing inoculant (Pioneer ${ }^{\circledR}$ 11GFT) on preservation and total tract digestibility of ensilage. In Proceedings of the XVth International Silage Conference, Madison, WI, USA, 27-29 July 2009.

56. Brüsemeister, F.; Kalzendorf, C.; Losand, B.; Ruser, B.; Thaysen, J. Influence of inoculating corn with a ferulate esterase releasing inoculant (Pioneer ${ }^{\circledR}$ brand 11CFT) on preservation and total tract digestibility of ensilage. In Proceedings of the XVth International Silage Conference, Madison, WI, USA, 27-29 July 2009.

57. Pitt, R.E.; Muck, R.E.; Pickering, N.B. A model of aerobic fungal growth in silage. 2. Aerobic stability. Grass Forage Sci. 1991, 46, 301-312. [CrossRef]

58. Middelhoven, W.J.; van Baalen, A.H.M. Development of the yeast flora of whole-crop maize during ensiling and during subsequent aerobiosis. J. Sci. Food Agric. 1988, 42, 199-207. [CrossRef]

59. Auerbach, H.; (International Silage Consultancy, Wettin-Löbejün, Germany); Theobald, P.; (University of Nürtingen-Geislingen, Nürtingen, Germany). Personal communication, 2018.

60. Auerbach, H.; Weber, U.; Weber, G.; Weiss, K.; Theobald, P. Effects of different chemical additives on the fermentation and aerobic stability of high-moisture corn ensiled in bags. In Proceedings of the XVIIth International Silage Conference, Piracicaba, Brazil, 1-3 July 2015.

61. Adesogan, A.T.; Salawu, M.B.; Ross, A.B.; Davies, D.R.; Brooks, A.E. Effect of Lactobacillus buchneri, Lactobacillus fermentum, Leuconostoc mesenteroides inoculants, or a chemical additive on the fermentation, aerobic stability, and nutritive value of crimped wheat grains. J. Dairy Sci. 2003, 86, 1789-1796. [CrossRef] 